\title{
Consideraciones en la elección de terapia anti-retroviral de primera línea en adultos
}

\author{
M. Elena Ceballos
}

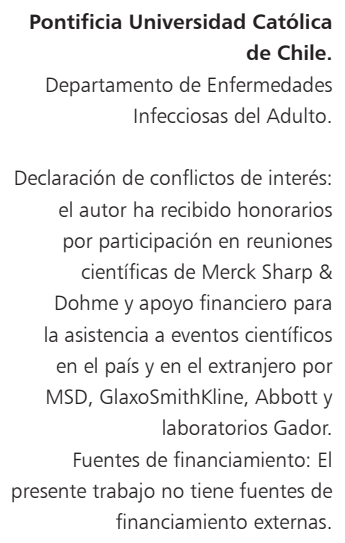

Pontificia Universidad Católica de Chile. Departamento de Enfermedades Infecciosas del Adulto.

Declaración de conflictos de interés: el autor ha recibido honorarios por participación en reuniones científicas de Merck Sharp \& Dohme y apoyo financiero para la asistencia a eventos científicos en el país y en el extranjero por MSD, GlaxoSmithKline, Abbott y laboratorios Gador.

Fuentes de financiamiento: El presente trabajo no tiene fuentes de financiamiento externas.

Recibido: 4 de marzo de 2013 Aceptado: 5 de agosto de 2013

Correspondencia a: María Elena Ceballos Valdivielso mceballos@med.puc.cl

\section{Introducción}

$\mathrm{L}$ a terapia anti-retroviral (TARV) ha disminuido significativamente la mortalidad de los pacientes con infección por el virus de la inmunodeficiencia humana/Síndrome de inmunodeficiencia adquirida (VIH/ SIDA) ${ }^{1}$.

Uno de los principales factores que influyen en el éxito de la TARV es la adherencia a ésta. La falta de adherencia conlleva a la resistencia a los fármacos anti-retrovirales (ARVs) y por ende, replicación viral persistente, con falla del tratamiento. Sin embargo, otros factores además de la adherencia del paciente también influirán en una adecuada respuesta a la TARV.

Una vez decidido que el paciente con infección por VIH tiene indicación de iniciar un esquema anti-retroviral (ARV), es fundamental evaluar cuál será la terapia más adecuada para ese paciente en particular.

Múltiples ARVs han sido aprobados para el manejo del paciente con infección por VIH; sin embargo, cada uno de ellos tiene indicaciones y contraindicaciones particulares que habrá que conocer antes de prescribir. Para esto se deberán considerar distintos factores como sexo, edad, ocupación, presencia de co-morbilidades, co-infecciones, hábitos, recuento basal de linfocitos T-CD4 y carga viral plasmática de VIH (Tabla 1). Se deberá explicar al paciente la forma de toma de la terapia, los horarios de administración, posibles interacciones medicamentosas, efectos adversos potenciales y necesidad de ajustar la dosis según la función renal o hepática.

Un adecuado tratamiento requerirá entonces, no sólo de la adherencia del paciente, sino también del compromiso del equipo de salud con el paciente, de escuchar sus inquietudes y educarlo en la forma correcta de uso de los ARVs.

Este artículo revisa los puntos anteriormente mencionados de una forma práctica, con el objetivo de guiar al médico que atiende pacientes con infección por VIH, en base a la evidencia actualmente disponible en el mundo.

Es importante recalcar que la evidencia científica es dinámica y, por ende, a futuro pudiesen existir variaciones en ciertas indicaciones, en base a nueva información disponible.

Tabla 1. Factores que influyen en la elección de la TARV

Sexo
Embarazo
Edad
Ocupación
Co-motbilidades médicas
Co-infecciones
Recuento basal de linfocitos T-CD4
Carga viral plasmática de VIH
Hábitos (uso de alcohol y drogas)
Potenciales efectos adversos
Interacciones medicamentosas
Función renal
Función hepática


Sexo

En general, no se observan diferencias de género en el pronóstico clínico (específicamente respuesta inmunológica y virológica) con el uso de los distintos ARVs. Sin embargo, existen ciertas consideraciones que debemos conocer principalmente relacionado con efectos adversos y el estado de embarazo en la mujer.

- En relación a posibles efectos adversos, hay dos principales consideraciones; la hepatotoxicidad con el uso de la nevirapina y las diferencias de género en las complicaciones metabólicas. En cuanto al uso de nevirapina, se debe evitar su indicación en mujeres con recuento de linfocitos T-CD4 > 250 céls/ $\mathrm{mm}^{3}$ y en hombres con recuento $>400$ céls $/ \mathrm{mm}^{3}$. Esto último, pues se ha visto una mayor incidencia de hepatotoxicidad en esos grupos específicos². En cuanto a las complicaciones metabólicas, algunos estudios han mostrado que, independientemente del fármaco utilizado, la mujer presenta un mayor aumento de la grasa central ${ }^{3}$, una mayor frecuencia de osteopenia y osteoporosis ${ }^{4}$ y una menor elevación de triglicéridos comparada con hombres que usan la misma TARV 5 .

- En cuanto al embarazo, la consideración principal es con el uso de efavirenz. Históricamente, el efavirenz ha estado contraindicado en mujeres en edad fértil y embarazadas, debido al riesgo de teratogenicidad de este medicamento conocido por estudios en animales. En estos ensayos, tres de 20 fetos/recién nacidos de monos, que recibieron efavirenz desde el día gestacional 20 a 150 , versus 0 de 20 controles presentaron malformaciones como anencefalia y microftalmia, entre otros ${ }^{6}$. Posteriormente, reportes de mujeres embarazadas expuestas a efavirenz en el primer trimestre del embarazo, mostraron incidencias de defectos de nacimiento similares a la población general ${ }^{7}$. Un meta-análisis posterior ${ }^{8}$ que incluyó nueve cohortes con reportes prospectivos de más de 1.000 mujeres expuestas a efavirenz en el primer trimestre del embarazo, no mostró un mayor riesgo de defectos de nacimiento entre las mujeres que recibieron efavirenz comparado con otros fármacos. Sin embargo, otro meta-análisis del Pediatric AIDS Clinical Trials Protocols (PACTG) 219 y $219 \mathrm{C}^{9}$ mostró una mayor frecuencia de defectos de nacimiento en expuestos a efavirenz durante el primer trimestre. Sin embargo, existen serios problemas metodológicos en este reporte que dificultan el análisis y posiblemente invalidan estos resultados.

En suma, faltan datos claros sobre el uso de efavirenz en el primer trimestre para poder determinar con claridad si efectivamente el riesgo de teratogenia de esta terapia es elevada.

Por otro lado, se ha observado que los cambios de te- rapia durante la gestación aumentan significativamente el riesgo de presentar supresión viral incompleta al final del embarazo. Debido a que el riesgo de generar defectos del desarrollo del tubo neural se restringe principalmente a las primeras cinco a seis semanas de gestación, período en que aún no es conocida la condición de embarazo, se recomienda en mujeres que ya estaban recibiendo efavirenz antes de la gestación, mantener la terapia para evitar una pérdida en el control viral y con ello un aumento en la transmisión viral al feto.

En resumen, y siguiendo las recomendaciones del panel de expertos de las guías perinatales del DHHS $2013^{10}$, se sugiere en mujeres en edad fértil y sexualmente activas, iniciar terapias distintas a efavirenz, mientras que en mujeres que se embarazan ya estando con este ARV, mantenerlo para evitar una pérdida del control virológico.

\section{Edad}

Al momento del diagnóstico, los pacientes con infección por VIH mayores de 50 años presentan mayor probabilidad de tener eventos definitorios de SIDA comparado con pacientes menores $(p=0,0001)$. Además, existe evidencia que muestra una recuperación significativamente más lenta en el recuento de linfocitos T-CD4 en pacientes mayores de 50 años ${ }^{11}$. Por esta razón, el ser mayor de 50 años es una indicación relativa para el inicio de TARV independiente del recuento de linfocitos T-CD4 basal. Por otro lado, a medida que avanza la edad, se van agregando co-morbilidades y con ellas el uso de fármacos, por lo que es importante evaluar en cada caso la mejor terapia para cada paciente. Para el caso de pacientes con osteoporosis, se recomendará usar un inhibidor nuclesídico de la transcriptasa reversa (INTR) distinto a tenofovir asociado a un inhibidor no nuclesídico de la transcriptasa reversa (INNTR) o a un inhibidor de integrasa (II), evitando el uso de inhibidores de proteasa (IP). En el caso de deterioro renal, se sugiere el uso de un INTR distinto a tenofovir, asociado a un INNTR. En pacientes con mayor riesgo cardiovascular o metabólico sería recomendable utilizar un INTR asociado a un IP no reforzado o a un II. (Ver sección co-morbilidades).

Otra consideración importante en pacientes mayores de 50 años es la polifarmacia y con ello el riesgo de interacciones. En general, los INNTR y los IP son los fármacos que presentan más interacciones debido a su metabolismo en el citocromo P450. (Ver sección interacciones). Por esta razón, una posible asociación en caso de pacientes con polifarmacia sería el uso de un INTR más un II. 


\section{Ocupación}

Un factor importante a considerar es la ocupación o tipo de trabajo al que se dedica el paciente. Un ejemplo son quienes trabajan con turnos nocturnos como personal de salud, guardias de seguridad, etc. En estos casos no se recomienda indicar efavirenz debido a que éste, dentro de sus efectos colaterales puede producir somnolencia, mareos, alteración de la concentración y confusión. Por esta razón, cuando se indica efavirenz, se sugiere su toma en la noche, previo a acostarse y en pacientes que realizan sus actividades laborales durante el día. Otras consideraciones son las estéticas. Un paciente que por su trabajo tenga contacto directo con público, habrá que evitar el uso de atazanavir dado la posibilidad de éste de generar ictericia. En el caso de un paciente con movilidad reducida se recomienda evitar el uso de lopinavir/ritonavir dada la posibilidad de presentar diarrea. En suma, es necesario conocer los efectos adversos más frecuentes de las terapias que indicamos, de manera de elegir la mejor terapia según cada caso particular (Ver sección efectos adversos).

\section{Co-morbillidades}

\section{Pacientes con alteración de la función renal}

Se recomienda evitar el uso de tenofovir como primera elección, debido a que se ha observado una disminución de la filtración glomerular aproximadamente en $1 \%$ de los pacientes ${ }^{12}$. La toxicidad renal se verá aumentada en caso de toxicidad renal previa, bajo peso corporal, edad avanzada, bajo recuento de linfocitos T-CD4 y uso simultáneo con otros nefrotóxicos ${ }^{13}$. Se ha observado una mayor toxicidad renal con la asociación de tenofovir a un IP con ritonavir, principalmente atazanavir/ritonavir y lopinavir/ritonavir versus la asociación de tenofovir a un INNTR $^{14,15}$.

Otro efecto adverso conocido es el síndrome de Fanconi, donde tenofovir se acumula en el interior de las células tubulares renales, generando toxicidad mitocondrial y eliminación tubular de proteínas, glucosa y fosfato. Este síndrome se describe en $0,1 \%$ de los $\operatorname{casos}^{13}$.

En el caso de utilizarlo en pacientes con alteración de la función renal, la dosis deberá ser ajustada según el clearance de creatinina. (Ver sección ajuste de dosis en insuficiencia renal).

Estudios recientes han mostrado nueva evidencia que refleja un efecto independiente de algunos IP como atazanavir/ritonavir y lopinavir/ritonavir sobre la alteración de la función renal, por lo que habría que utilizar con cautela estos ARVs en pacientes con falla renal conocida o elegir alternativas ${ }^{16}$.

\section{Pacientes con osteoporosis o fracturas patológicas}

Considerar alternativas al tenofovir y a los IP, pues éstos se asocian a mayor pérdida de masa ósea. El estudio
ASSERT comparó los cambios en la densidad mineral ósea entre pacientes con infección por VIH vírgenes a terapia. Un grupo recibió abacavir/lamivudina y otro grupo, tenofovir/emtricitabina, ambos en combinación con efavirenz como tercer fármaco. Se observó una diferencia estadísticamente significativa en la caída de la densidad mineral ósea en el grupo de tenofovir sobre abacavir en caderas y columna lumbar ${ }^{17}$.

En cuanto a los IP, Divivier mostró que existía una significativa mayor pérdida de densidad mineral ósea en la columna lumbar en pacientes usuarios de IP comparado con otros esquemas terapéuticos ${ }^{18}$.

\section{Pacientes con citopenia previo al inicio de la TARV}

Se recomienda iniciar terapia con un INTR distinto a zidovudina, debido a su conocido efecto citopénico sobre la médula ósea, principalmente durante las primeras semanas de tratamiento.

\section{Pacientes con factores de riesgo cardiovascular}

Durante algunos años, la exposición a abacavir ha sido considerada un factor de riesgo para presentar eventos cardiovasculares $^{19}$. Recientemente, un meta-análisis ${ }^{20}$ mostró la falta de asociación de esta terapia con un mayor riesgo de infarto agudo al miocardio (RR 0,73; IC 95\% $0,39-1,35 ; \mathrm{p}=0,31)$, eventos cardiovasculares mayores (RR 0,95; IC 0,62-1,44; $\mathrm{p}=0,80$ ) y mortalidad global (RR 1,20; IC 95\% 0,63-2,27; p =0,58). En contraste, en otro estudio observacional, de Veterans Affairs Study, con 11.000 pacientes (hombres en su mayoría), el abacavir se asoció a un aumento de riesgo de eventos cardiovascula$\mathrm{res}^{21}$. En resumen, la evidencia es aún contradictoria, por lo que habrá que evaluar la indicación con precaución y según cada paciente en forma individual.

\section{Pacientes con alteraciones metabólicas}

Las TARVs pueden producir un aumento significativo de los niveles de lípidos, principalmente de triglicéridos, e intolerancia a la glucosa. Esto se ha observado en forma más evidente con los IP, especialmente cuando son reforzados con ritonavir. Las combinaciones que más producen dislipidemia son lopinavir/ritonavir y fosamprenavir/ ritonavir. En menor medida se observa dislipidemia con atazanavir/ritonavir y darunavir/ritonavir. También se observa hipertrigliceridemia con el uso de efavirenz. En casos de dislipidemia grave o de difícil manejo, o en pacientes con muchos factores de riesgo cardiovascular, se podría considerar el uso de un IP con menor efecto sobre los lípidos, en ciertos casos específicos un IP no reforzado o nuevos fármacos como los II, estos últimos debido a la baja toxicidad metabólica descrita ${ }^{22}$.

\section{Pacientes con patología psiquiátrica}

Debido a que el efavirenz genera riesgo de exacerbar una depresión e ideas suicidas, se sugiere evitar este 
INNTR en pacientes con co-morbilidades psiquiátricas pre-existentes.

\section{Co-infecciones}

\section{Coinfección VIH/VHB}

En pacientes portadores crónicos de hepatitis B (HB$\mathrm{sAg}+$ ) co-infectados con VIH está indicada la TARV, independiente del recuento de linfocitos T-CD4, debido a que se ha demostrado que esta co-infección confiere una progresión más rápida a cirrosis, daño hepático crónico y hepatocarcinoma, comparado con pacientes sin VIH. El tratamiento indicado en estos casos es una terapia biasociada para el virus de la hepatitis B con tenofovir + lamivudina o emtricitabina (que a la vez tendrían efecto contra el VIH) asociado a un tercer fármaco con acción para el VIH. En caso de contraindicación a tenofovir, la alternativa preferida es el uso de entecavir, en asociación a una triple TARV. Otras alternativas son peg-interferón alfa como monoterapia o adefovir asociado a lamivudina o emtricitabina, junto con la TARV ${ }^{23}$.

\section{Coinfección VIH/VHC}

En el caso de pacientes con co-infección VIH/virus hepatitis $\mathrm{C}$ (VHC), la progresión a cirrosis y descompensación de la enfermedad hepática es mayor comparada con pacientes portadores de $\mathrm{VHC} \sin \mathrm{VIH}^{24}$, especialmente en individuos con CD4 $\leq$ a 350 céls $/ \mathrm{mm}^{3}$. La TARV podría retrasar la progresión de la enfermedad hepática al disminuir la inflamación crónica y restaurar la función inmune. Por esto, para la mayoría de los pacientes co-infectados debe indicarse TARV. Por otro lado, con la evidencia actual, distintas sociedades científicas como el National Institutes of Health, US Public Health Service, American Association for the Study of Liver Diseases ${ }^{25}$, Infectious Diseases Society of America, European Consensus Conference Panel ${ }^{26}$, European AIDS Clinical Society ${ }^{27}$ y el US Department of Health and Human Services ${ }^{23}$ sugieren considerar el tratamiento para VHC en pacientes co-infectados con VIH.

Debido a las importantes interacciones medicamentosas y sobreposición de toxicidades que existen entre la TARV y la terapia para el VHC, se recomienda iniciar TARV antes de iniciar el tratamiento para VHC, en pacientes con inmunosupresión intensa (CD4 menor a 200 céls $/ \mathrm{mm}^{3}$ ) o en aquellos con mínima fibrosis. Por otro lado, en pacientes con CD4 mayores a 500 céls $/ \mathrm{mm}^{3}$, la TARV podría diferirse hasta completar el tratamiento para $\mathrm{VHC}^{23}$.

Las terapias para VHC actualmente en uso son peginterferón/ribavirina, éstas asociadas a un IP NS3/4A para VHC (boceprevir o telaprevir) en los casos de pacientes con VHC genotipo 1.

Estas terapias presentan múltiples interacciones con la TARV, especialmente los IP contra el VHC. En el caso de necesitarse superponer terapias, la TARV deberá ser cuidadosamente seleccionada o modificada para disminuir las interacciones y toxicidades.

En el apartado de interacciones se encuentran los fármacos que no se deberán asociar, así como las alternativas terapéuticas en los casos de necesitarse realizar tratamiento simultáneo. De todos modos, los esquemas terapéuticos para el VHC se encuentran en constante avance. Actualmente están en estudio regímenes libres de interferón; (nuevos IP para VHC asociados a inhibidores de polimerasa, con o sin ribavirina) con resultados alentadores. Estos resultados podrían generar cambios en la indicación de la oportunidad de inicio de la TARV ${ }^{28-31}$.

\section{Coinfección VIH/tuberculosis}

En pacientes con tuberculosis activa, se deben considerar las interacciones que presentan los IP, INNTR, II y antagonistas de co-receptor CCR5 con las rifamicinas utilizadas en el tratamiento de la TBC. Las rifamicinas (rifampicina y rifabutina) son inductoras de la enzima CYP450 por lo que se deberá ajustar las dosis del ARV en algunos casos y en otros casos no asociar, como es el caso de los IP con rifampicina y considerar fármacos alternativos. (Ver: interacciones).

\section{Recuento de linfocitos CD4 y carga viral plasmática de VIH}

Se han realizado distintos estudios para comparar la eficacia virológica e inmunológica entre las TARV. A continuación, comentaremos algunos de los estudios más significativos sobre este tema, separados por familias de ARVs, que nos pueden ayudar en la elección de la terapia.

\section{Inhibidores nucleosídicos de la transcriptasa reversa}

En cuanto a recuperación inmunológica, un estudio publicado el año 2004, mostró que el uso de abacavir (en combinación con lamivudina y efavirenz) generaba un mayor aumento de linfocitos T-CD4 $(p=0,005)$ a las 24 y 48 semanas de iniciada la terapia comparado con zidovudina (en combinación con lamivudina y efavirenz), y una similar respuesta virológica ${ }^{32}$.

El estudio Gilead 934, publicado el año 2006, mostró que con el uso de tenofovir/emtricitabina comparado con zidovudina/lamivudina (ambos en asociación con efavirenz), una mayor cantidad de pacientes alcanzaba carga viral de $\mathrm{VIH}<400$ copias de $\mathrm{ARN} / \mathrm{ml}(75 \%$ el grupo tenofovir vs $62 \%$ grupo lamivudina, $\mathrm{p}=0,004)$ a las 96 semanas de terapia. Para el objetivo de alcanzar $<$ de 50 copias/ml, también se observó una mejor respuesta, pero no estadísticamente significativa ( 67 vs $61 \% ; p=0,16)^{33}$. 
El estudio ACTG $5202^{34}$ comparó tenofovir/emtricitabina versus abacavir/lamivudina asociados a efavirenz o atazanavir/ritonavir en pacientes vírgenes a tratamiento. Se observó que en pacientes con carga viral basal $>100.000$ copias de ARN viral/ml, el tiempo en alcanzar falla virológica fue menor en el grupo que usó abacavir/ lamivudina versus tenofovir/emtricitabina, independiente de la terapia utilizada (efavirenz o atazanavir/ritonavir).

Estos hallazgos no fueron confirmados en el estudio HEAT $^{35}$, donde, manteniendo la comparación tenofovir/ emtricitabina y abacavir/lamivudina (y utilizando lopinavir/ritonavir como tercer fármaco) se observó similar eficacia virológica e inmunológica, independiente de si la carga viral basal de VIH fuese $>$ o $<$ a 100.000 copias ARN/ml.

Por lo tanto, en la elección de los INTR, disponemos de tenofovir/lamivudina o abacavir/lamivudina, los cuales serían similares en cuanto a recuperación inmunológica y en eficacia virológica, con la excepción de pacientes que basalmente presentan cargas virales altas, donde sería preferible iniciar tenofovir sobre abacavir. Si bien la asociación zidovudina/lamivudina ha mostrado buenos resultados a lo largo de las últimas décadas, actualmente está siendo reemplazada por tenofovir y abacavir dado sus mejores resultados en eficacia inmuno-virológica.

Inhibidores no nucleosídicos de la transcriptasa reversa

El estudio ACTG $5142^{36}$ compara tres regímenes iniciales en pacientes vírgenes a terapia: un grupo efavirenz (asociado a zidovudina/lamivudina o stavudina/ lamivudina o tenofovir/lamivudina), otro grupo lopinavir/ ritonavir (asociado a zidovudina/lamivudina o stavudina/ lamivudina o tenofovir/lamivudina) y un tercer grupo que asocia efavirenz con lopinavir/ritonavir. De los resultados se observa que un mayor porcentaje de pacientes en el grupo efavirenz asociado a un INTR alcanza a las 96 semanas una carga viral de $\mathrm{VIH}<200$ copias de ARN/ $\mathrm{ml}(\mathrm{p}=0,04) \mathrm{y}<50$ copias de ARN/ml $(\mathrm{p}=0,003)$ al compararlo con el grupo lopinavir/ritonavir asociado a un INTR. Por otro lado, se observa un mayor aumento de linfocitos T-CD4 desde el basal a la semana 96 en el grupo lopinavir/ritonavir (asociado a INTR o a efavirenz) comparado con el grupo efavirenz más INTR.

Más antiguo es el estudio $2 \mathrm{NN}^{37}$, donde se compara efavirenz con nevirapina. Si bien se observó similar respuesta virológica entre ambos fármacos, el grupo con nevirapina no alcanzó la no inferioridad debido a su mayor toxicidad en relación al grupo control.

Rilpivirina (INNTR no disponible en nuestro país), ha demostrado similar eficacia virológica comparada con efavirenz en pacientes vírgenes a tratamiento (ambas combinadas con tenofovir/emtricitabina), pero sólo en el grupo de pacientes con carga viral basal de $\mathrm{VIH}<100.000$ copias de $\mathrm{ARN} / \mathrm{ml}^{38}$.
En el caso de etravirina, este INNTR demostró su eficacia virológica inicialmente en pacientes experimentados a TARV. El estudio incluyó pacientes con un régimen optimizado que incluía darunavir/ritonavir y los separó en dos grupos; un grupo al cual se le asoció etravirina y otro grupo a placebo. El grupo con etravirina demostró superioridad en supresión virológica (carga viral de VIH $<50$ copias $/ \mathrm{ml}$ ) y en pronósticos clínicos como nuevos eventos definitorios de SIDA y muerte. Estos resultados se mantuvieron a las 48 semanas de seguimiento ${ }^{39}$.

Posteriormente, en el estudio $\mathrm{SENSE}^{40}$, se comparó etravirina $400 \mathrm{mg}$ al día con efavirenz $600 \mathrm{mg}$ al día, cada uno asociado a dos INTR, en pacientes vírgenes a TARV. Si bien fue un estudio randomizado, sólo se incluyeron 157 pacientes. El objetivo primario fue observar la aparición de eventos neuro-psiquiátricos y el secundario, evaluar la respuesta virológica. A las 48 semanas, etravirina mostró no inferioridad ante efavirenz en cuanto a eficacia virológica y se observaron significativamente menos efectos adversos neuropsiquiátricos en el grupo con etravirina $(6,3 \%)$ versus efavirenz $(21,4 \%) p=0,001$. Si bien se muestra que etravirina podría ser una opción de primera línea, por ahora su precio y los escasos datos en pacientes vírgenes a TARV desaconsejan su uso.

En suma, para la elección de un INNTR como tercer fármaco en primera línea, tenemos dos alternativas en nuestro país. Por eficacia y seguridad el efavirenz es el medicamento de elección; sin embargo, nevirapina podrá ser utilizada con precaución, observando de cerca su toxicidad.

\section{Inhibidores de proteasa}

El estudio CASTLE ${ }^{41}$ fue un estudio de no inferioridad que comparó atazanavir/ritonavir con lopinavir/ritonavir, cada uno asociado a tenofovir/emtricitabina. Se observó una similar eficacia virológica a la semana 48. La eficacia inmunológica fue similar entre ambos grupos, excepto para los pacientes que presentaban menos de 50 linfocitos T-CD4 en forma basal, donde el ascenso fue menor en el grupo lopinavir/ritonavir. El seguimiento a la semana 96, en un análisis por intención de tratar, mostró la no inferioridad de atazanavir sobre el lopinavir, donde un mayor porcentaje de pacientes en el grupo atazanavir/ritonavir mantenía carga viral VIH $<50$ copias/ml. $(\mathrm{p}<0,05)^{42}$.

El estudio ALERT $^{43}$ comparó atazanavir/ritonavir con fosamprenavir/ritonavir, ambos asociados a tenofovir/ emtricitabina. No se observaron diferencias en cuanto a eficacia inmunológica ni virológica entre los grupos a la semana 48. Un mayor porcentaje de pacientes en el grupo con fosamprenavir presentó diarrea e hipertrigliceridemia.

El estudio KLEAN ${ }^{44}$ comparó lopinavir/ritonavir con fosamprenavir/ritonavir, ambos asociados a abacavir/ lamivudina. Se observó una similar respuesta virológica e inmunológica a las 48 semanas. Esta respuesta se man- 
tuvo a las 144 semanas de seguimiento ${ }^{45}$. El aumento de triglicéridos fue de aproximadamente $8 \%$, similar para ambos grupos de terapia.

El estudio GEMINI ${ }^{46}$ comparó la utilización de saquinavir/ritonavir con lopinavir/ritonavir, ambos con tenofovir/emtricitabina. El grupo con saquinavir/ ritonavir mostró no inferioridad al grupo con lopinavir/ ritonavir a las 48 semanas en respuesta virológica e inmunológica; sin embargo, en el grupo con saquinavir se observó más trastornos del intervalo QT, mientras que en el grupo con lopinavir, mayor frecuencia de hipertrigliceridemia.

El estudio ARTEMIS ${ }^{47}$, comparó lopinavir/ritonavir con darunavir/ritonavir, ambos en combinación con tenofovir/emtricitabina. En este caso, el grupo con darunavir/ ritonavir mostró no inferioridad a lopinavir/ritonavir a las 48 semanas, exceptuando al subgrupo con carga viral $>100.000$ copias de ARN/ml, donde se observó una menor respuesta virológica en el grupo lopinavir $(<0,05)$. A las 96 semanas del estudio, el grupo darunavir alcanzó a mostrar superioridad al grupo lopinavir en cuanto a respuesta virológica.

Con estos estudios podemos observar que en la elección de un IP como tercer fármaco, en general no hay grandes diferencias entre atazanavir, lopinavir o fosamprenavir, cada uno de ellos reforzado con ritonavir. En cuanto a eficacia inmunológica o virológica, cualquiera de ellas sería una buena opción en primera línea. Sin embargo, dado sus efectos adversos en el metabolismo, fosamprenavir/ritonavir y lopinavir/ritonavir se mantienen como terapias alternativas, mientras que atazanavir/ritonavir como preferida ${ }^{23}$. En cuanto al darunavir/ritonavir, si bien ha mostrado excelente eficacia virológica, en nuestro país, la guía del año 2009 lo incluye como terapia de rescate, dado su eficacia contra virus resistentes a otros $\mathrm{IP}^{48}$.

\section{Inhibidores de integrasa}

Para los fármacos más nuevos, el estudio START$\mathrm{MRK}^{49}$, comparó el uso de raltegravir con efavirenz, asociados cada uno a tenofovir/emtricitabina, en pacientes vírgenes a terapia. En este estudio se observó una similar eficacia virológica entre ambos grupos a las 156 semanas de seguimiento; sin embargo, en el seguimiento a 240 semanas se observó superioridad de raltegravir en la supresión virológica $<50$ copias de ARN/ml (71 vs 61\%; diferencia de 9,5 (IC 95\% 1,7-17,3). En los pacientes en el grupo raltegravir se observaron menores efectos adversos relacionados al fármaco, además de un perfil lipídico más favorable.

Nuevos estudios han mostrado la eficacia de elvitegravir/cobicistat/ tenofovir/emtricitabina, comparado con distintos esquemas terapéuticos. Rockstrohet y cols. ${ }^{50}$ compararon este esquema con tenofovir/emtricitabina/ atazanavir/ritonavir. El nuevo esquema mostró evidencias de no inferioridad en cuanto al éxito virológico a las 48 y 96 semanas, al igual que similares resultados en seguridad. Zolopa y cols. ${ }^{51}$ compararon este esquema con tenofovir/ emtricitabina/efavirenz. A las 48 y 96 semanas elvitegravir/cobicistat/ tenofovir/emtricitabina fue no inferior a su grupo comparador y no se observaron diferencias significativas en seguridad.

Otra alternativa a utilizar como tercer fármaco es un II como raltegravir. Se considera especialmente para pacientes con contraindicaciones a efavirenz y a IP o en aquellos con trastornos metabólicos. Actualmente elvitegravir no se encuentra disponible en nuestro país.

\section{Antagonistas de correceptor CCR5}

El estudio MERIT comparó efavirenz versus maraviroc (ambos en combinación con zidovudina/lamivudina). En un post análisis, utilizando un estudio de tropismo más sensible que el utilizado en el estudio inicial, se observaron similares respuestas virológicas entre ambos grupos ${ }^{52}$.

Por ahora, dado que los datos son menos evidentes para maraviroc en primera línea, no se recomienda como tercer fármaco preferido sino que se mantiene sólo para situaciones de rescate.

\section{Consideraciones en pacientes drogadictos y alcohólicos}

Aproximadamente $28 \%$ de las personas con infección por VIH en los E.U.A son usuarios de drogas ilícitas ${ }^{53}$. En la cohorte de pacientes con VIH de la Pontificia Universidad Católica, $12 \%$ consume alcohol en altas cantidades y $12 \%$ es usuario de drogas, siendo las más frecuentes la marihuana y cocaína ${ }^{54}$.

Si bien el tratamiento del VIH puede ser exitoso en usuarios de drogas ilícitas y alcohol, el cuidado de estos pacientes presenta desafíos importantes.

Múltiples estudios muestran que el consumidor de alcohol o drogas tiene una mayor posibilidad de no recibir TARV, esto por distintos factores como el menor compromiso con los cuidados de salud y una menor adherencia a la TARV ${ }^{55}$. Además se observa una mayor asociación a co-morbilidades psiquiátricas como son la depresión y ansiedad, lo cual es un predictor importante de pobre adherencia y mal pronóstico terapéutico ${ }^{23}$.

El consumo de drogas y alcohol impacta negativamente en la progresión del VIH, tanto por la disminución del recuento de linfocitos T-CD4 como por la no supresión de la carga viral $^{56-58}$. Hay evidencias que el consumo crónico de alcohol aumenta la susceptibilidad a ciertas enfermedades infecciosas (como tuberculosis y neumonías bacterianas), la gravedad de las hepatitis virales y el riesgo de cáncer hepatocelular. Además, estas enfermedades pueden verse aceleradas por el déficit vitamínico y la malnutrición asociada al etilismo ${ }^{55}$. 
Por otra parte, el consumo de alcohol y ciertas drogas puede afectar el metabolismo de algunos ARVs, especialmente los IP, metabolizados por el citocromo P450. El abuso crónico de alcohol aumenta el riesgo de presentar toxicidad a la terapia e ineficacia en la respuesta terapéutica de ciertos ARVs por una inadecuada concentración plasmática de estos ${ }^{55}$. Será fundamental evaluar las interacciones entre ARVs y drogas de abuso que pueda utilizar el paciente, así como también aquellas utilizadas como tratamiento de dependencia a opioides como son la metadona o buprenorfina (Ver: interacciones).

Será entonces muy importante la selección del ARV, cuidando las interacciones posibles entre drogas ilícitas y ARVs, incluido los efectos adversos y toxicidades. Además se deberá evaluar la indicación de regímenes simples considerando una mejor adherencia a la TAR.

La valoración de los pacientes alcohólicos o consumidores de drogas debe ser parte rutinaria de la historia clínica, realizándose de una manera profesional, directa y sin juzgar al paciente. Deberá existir una colaboración cercana con programas de tratamiento de abuso de sustancias, de manera de lograr un exitoso manejo de la infección por $\mathrm{VIH}^{23}$.

\section{Potenciales efectos adversos}

Si bien las TARV son cada vez menos tóxicas, se describe aproximadamente $10 \%$ de efectos adversos, tanto precoces como tardíos, secundarios al uso de TARV. Algunos de éstos presentan la gravedad suficiente para necesitar cambiar una terapia. En la cohorte chilena de SIDA $^{59}, 52 \%$ requirió un cambio del primer esquema de terapia y dentro de éstos, $44,9 \%$ fue por toxicidad. En la cohorte de pacientes VIH- UC ${ }^{54}, 42 \%$ cambió su primer esquema de terapia, siendo $65 \%$ de estos cambios causado por toxicidad. Se describen los principales efectos adversos a considerar según los ARVs más frecuentemente usados (Tabla 2).

\section{Potenciales interacciones}

Otro elemento a considerar previo a la elección de la TAR, son las posibles interacciones medicamentosas. Son múltiples las interacciones que existen entre las TARV y otros fármacos. Esto porque tanto la familia de los INNTR como la de los IP son metabolizados por el citocromo P450 hepático, al igual que muchos medicamentos. Esta interacción puede significar un aumento o una disminución de los niveles plasmáticos de alguno de los fármacos. Es fundamental revisar las terapias concomitantes que utilizan nuestros pacientes, así como explicarles a ellos que para cualquier nuevo medicamento que les sea prescrito, deberán ser revisadas sus interacciones, de manera de evitar fallas terapéuticas o por otro lado, toxicidades.

Algunas de las interacciones entre la TARV y otros fármacos, con las que los médicos nos vemos frecuentemente enfrentados son algunos antimicrobianos, antiácidos, anticonvulsivantes, anticonceptivos, corticosteroides inhalatorios, benzodiazepinas, entre otros (Tabla 3).

\section{Antimicrobianos}

Dentro de los antimicrobianos, claritromicina presenta interacción con la familia de los IP y de los INNTR. La asociación con INNTR genera una disminución del área bajo la curva (AUC) de claritromicina entre 31 y 39\% según el no nucleósido utilizado, por lo que se sugiere considerar una alternativa, como azitromicina. En el caso de la asociación con IP, aumenta en forma importante el AUC de claritromicina (hasta en $94 \%$ en la asociación con atazanavir/ritonavir), pudiendo incluso generar prolongación del QT. Por esta razón, según el IP que use el paciente se sugiere reducir las dosis de claritromicina según la velocidad de filtración glomerular o en otros casos evitar la claritromicina y utilizar un macrólido alternativo como azitromicina.

\section{Tratamiento antituberculoso}

En cuanto al uso de fármacos antituberculosos, considerar las interacciones que presentan los IP, INNTR, los II y antagonistas de co-receptor CCR5 con las rifamicinas utilizadas en el tratamiento de la tuberculosis. Las rifamicinas (rifampicina y rifabutina) son inductores de la enzima CYP450. Rifampicina aumenta el aclaramiento de los INNTR efavirenz y nevirapina. La asociación efavirenz-rifampicina reduce la exposición a efavirenz en 20-25\%; sin embargo, el éxito virológico no se ve afectado. Algunos expertos recomiendan aumentar las dosis de efavirenz de 600 a $800 \mathrm{mg}$ al día, pero los datos clínicos, inmunológicos y virológicos de estudios observacionales no apoyan esta práctica. De hecho, en pacientes tratados con dosis habituales de efavirenz mientras toman rifampicina no se ha observado fallas en el tratamiento ${ }^{23,60}$. Esto no ha sido demostrado con nevirapina, donde la rifampicina disminuye la exposición de nevirapina en $50 \%$, con una consecuente falla virológica ${ }^{60}$.

Mucho mayor es el efecto de la co-administración de rifampicina con IP, donde su asociación resulta en una disminución de 80-98\% de la exposición al IP, comprometiendo la efectividad de la TARV.

Algunos estudios han planteado la posibilidad de evitar este efecto mediante el aumento de dosis de ritonavir a $400 \mathrm{mg}$ cada $12 \mathrm{~h}$ o lopinavir/ritonavir $800 / 200 \mathrm{mg}$, cada $12 \mathrm{~h}$ (4 tabletas cada $12 \mathrm{~h}$ ). Sin embargo, faltan datos sobre eficacia, además del aumento de toxicidad que esto podría generar, por lo que el aumento a la doble dosis de IP no es por ahora la alternativa más aceptada ${ }^{61}$. 


\begin{tabular}{|c|c|c|}
\hline Terapia antirretroviral & Principales efectos adversos & Comentarios \\
\hline Zidovudina & $\begin{array}{l}\text { - Anemia, neutropenia } \\
\text { - Acidosis láctica } \\
\text { - Lipoatrofia } \\
\text { - Náuseas, vómitos }\end{array}$ & $\begin{array}{l}\text { Presentación de la anemia entre la segunda y la cuarta semana, neutro- } \\
\text { penia entre la sexta y la octava semana }\end{array}$ \\
\hline Lamivudina & $\begin{array}{l}\text { Exacerbación de hepatitis en co-infectados por VHB que suspenden } \\
\text { lamivudina }\end{array}$ & \\
\hline Abacavir & $\begin{array}{l}\text { Síndrome de hipersensibilidad. (Los síntomas empeoran con la continua- } \\
\text { ción de fármaco, suspender de inmediato) }\end{array}$ & $\begin{array}{l}\text { Promedio de inicio de reacción a los } 9 \text { días de su introducción, } 90 \% \text { de } \\
\text { las reacciones aparecen durante las primeras seis semanas de uso }\end{array}$ \\
\hline Tenofovir & $\begin{array}{l}\text { - Nefrotoxicidad: aumento de creatininemia, síndrome de Fanconi } \\
\text { - Osteoporosis/osteopenia } \\
\text { - Exacerbación de hepatitis en co-infectados por VHB que suspenden } \\
\text { tenofovir }\end{array}$ & $\begin{array}{l}\text { Disminución de densidad mineral y de la función renal es mayor con la } \\
\text { asociación a IP }\end{array}$ \\
\hline Efavirenz & $\begin{array}{l}\text { - Somnolencia, mareos, sueños vividos, alteración de la concentración. } \\
\text { Podría exacerbar depresión, sicosis e ideas suicidas } \\
\text { - Rash } \\
\text { - Teratogenia demostrada en primates y potencial en humanos }\end{array}$ & $\begin{array}{l}\text { Efectos neuropsiquiátricos ocurren a los pocos días de su introducción, } \\
\text { pero ceden en general entre la segunda y cuarta semana de uso }\end{array}$ \\
\hline Nevirapina & $\begin{array}{l}\text { - Hepatotoxicidad: riesgo es mayor para pacientes vírgenes a trata- } \\
\text { miento, especialmente mujeres con linfocitos CD } 4 \geq 250 \text { céls } / \mathrm{mm}^{3} \text { y } \\
\text { hombres con linfocitos } C D 4 \geq 400 \text { céls } / \mathrm{mm}^{3} \\
\text { - Rash, incluído síndrome de Stevens Johnson }\end{array}$ & Riesgo es mayor en los primeros meses de tratamiento \\
\hline Etravirina & $\begin{array}{l}\text { - Rash cutáneo (incluido riesgo de Stevens Johnson) } \\
\text { - Síndrome de hipersensibilidad }\end{array}$ & \\
\hline Lopinavir/ritonavir & $\begin{array}{l}\text { - Intolerancia gastrointestinal (diarrea, náuseas y vómitos) } \\
\text { - Dislipidemia (especialmente hipertrigliceridemia) } \\
\text { - Insulino resistencia, diabetes mellitus } \\
\text { - Mal distribución grasa } \\
\text { - Pancreatitis } \\
\text { - Prolongación del intervalo PR y QT }\end{array}$ & Efectos metabólicos exacerbados por el uso concomitante de ritonavir \\
\hline Atazanavir & $\begin{array}{l}\text { - Hiperbilirrubinemia indirecta } \rightarrow \text { ictericia } \\
\text { - Hiperglicemia/dislipidemia } \\
\text { - Nefrolitiasis } \\
\text { - Mal distribución grasa } \\
\text { - Prolongación del intervalo PR }\end{array}$ & $\begin{array}{l}\text { En la cohorte VIH-UC } 64 \% \text { de los pacientes con atazanavir desarrollan } \\
\text { hiperbilirubinemia. Sin embargo, sólo } 5 \% \text { aproximadamente debe cam- } \\
\text { biar de terapia por presentar ictericia clínica }\end{array}$ \\
\hline Fosamprenavir & $\begin{array}{l}\text { - Rash cutáneo } \\
\text { - Hiperglicemia/dislipidemia } \\
\text { - Hepatotoxicidad } \\
\text { - Mal distribución grasa }\end{array}$ & \\
\hline Darunavir & $\begin{array}{l}\text { - Rash cutáneo (incluido riesgo de Stevens Johnson) } \\
\text { - Hiperglicemia/dislipidemia } \\
\text { - Hepatotoxicidad } \\
\text { - Mal distribución grasa }\end{array}$ & \\
\hline Raltegravir & $\begin{array}{l}\text { - Rash cutáneo (incluido riesgo de Stevens Johnson) } \\
\text { - Molestias gastrointestinales } \\
\text { - Elevación de CPK, debilidad muscular hasta rabdomiolisis }\end{array}$ & \\
\hline Maraviroc & $\begin{array}{l}\text { - Dolor abdominal, pirexia } \\
\text { - Rash } \\
\text { - Tos } \\
\text { - Infecciones respiratorias altas } \\
\text { - Hepatotoxicidad } \\
\text { - Hipotensión ortostática }\end{array}$ & \\
\hline
\end{tabular}




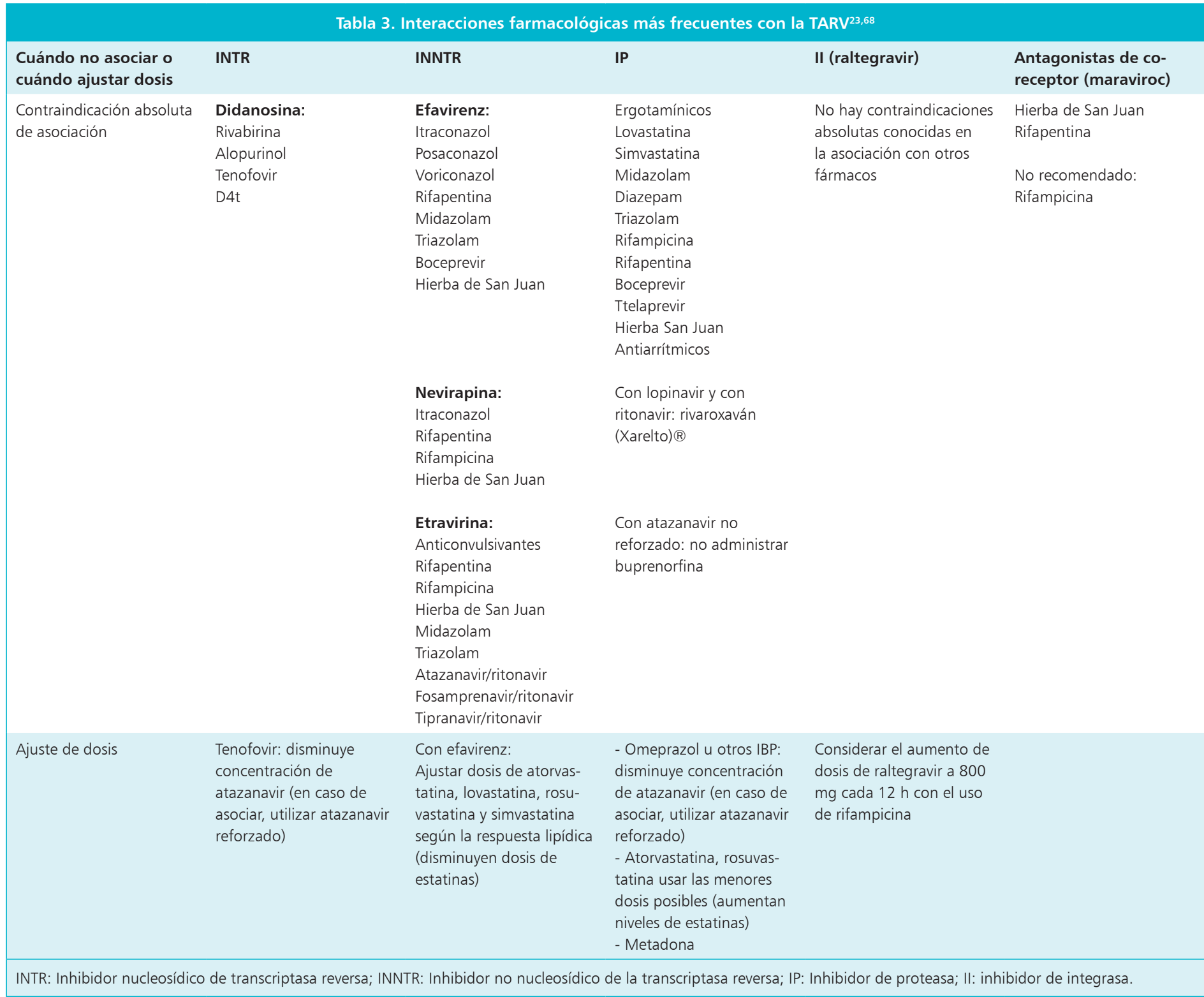

En estos casos, la alternativa preferida para asociar con IP reforzados es rifabutina, dado que ésta en un inductor débil de la enzima CYP450. Sin embargo, se deberá tomar en cuenta que esta rifamicina aumenta su concentración en exposición a IP, pudiendo generar toxicidad; por lo tanto, la dosis de rifabutina deberá ser ajustada a $150 \mathrm{mg}$ al día o $300 \mathrm{mg}$ día por medio ${ }^{60}$.

Para el caso del raltegravir, la rifamicina con menor interacción es rifabutina. Esta última reduce las concentraciones de raltegravir; sin embargo, esto no afecta su acción en términos de eficacia virológica. En caso de no disponer de rifabutina, es posible utilizar rifampicina, pero dado que ésta disminuye los niveles del II, se deberá doblar la dosis de raltegravir a $800 \mathrm{mg}$ cada $12 \mathrm{~h}$ y realizar un monitoreo estricto de la respuesta virológica ${ }^{62}$.

Dado que rifabutina no se encuentra disponible en nuestro país, en nuestros pacientes utilizamos la opción de rifampicina en asociación con efavirenz o raltegravir si el paciente no es candidato a efavirenz.

\section{Tratamiento para $\mathrm{VHC}$}

Anteriormente comentamos que existen importantes interacciones entre las terapias para VIH y VHC. Algunos ejemplos de interacciones son didanosina con ribavirina (no asociar debido a la alta toxicidad mitocondrial), zidovudina con ribavirina (evitar asociación debido a un alto 
riesgo de anemia), boceprevir con atazanavir/ritonavir, darunavir/ritonavir, lopinavir/ritonavir o efavirenz (no se recomienda su asociación por la alta tasa de interacciones) y la asociación de telaprevir con darunavir/ritonavir, fosamprenavir/ritonavir o lopinavir/ritonavir (evitar su asociación por la alta tasa de interacciones). Algunas opciones terapéuticas serían utilizar dos INTR donde no se incluya zidovudina ni didanosina asociado a efavirenz o atazanavir/ritonavir (en estos casos el IP elegido para VHC deberá ser telaprevir y no boceprevir) o dos INTR asociados a raltegravir (acá se podrá utilizar boceprevir o telaprevir contra $\mathrm{VHC})^{23}$.

\section{Benzodiazepinas}

Otros medicamentos frecuentemente utilizados son las benzodiazepinas. Se deberá evitar la co-administración de efavirenz con midazolam oral y triazolam por el aumento de las concentraciones de estos sedantes. Es posible utilizar alprazolam y lorazepam en asociación a efavirenz sin ajuste de dosis, monitoreando los efectos clínicos de las benzodiazepinas. Las interacciones con los IP son mayores. Está contraindicada la asociación entre cualquier IP con midazolam oral y triazolam. En cuanto a alprazolam y diazepam, sus concentraciones plasmáticas aumentan al co-administrar con cualquier IP. Se sugiere utilizar la alternativa de lorazepam debido a que tendría la menor interacción con los IP entre la familia de las benzodiazepinas.

\section{Corticosteroides inhalatorios}

Respecto al uso de corticosteroides inhalatorios, se deberá evitar el uso de budesonida y fluticasona inhalada o intranasal con todos los IP reforzados con ritonavir. Esto debido a que ritonavir aumenta en forma importante las concentraciones plasmáticas de estos corticosteroides, pudiendo generar incluso un síndrome de Cushing. Se deberá tener precaución y evitar la co-administración de lopinavir/ritonavir con prednisona oral, ya que esta asociación aumenta los niveles del corticoesteroide y disminuye los niveles plasmáticos de lopinavir.

\section{Ergotamínicos}

Es importante tener clara la contraindicación absoluta de administrar derivados del ergot (vasoconstrictores), medicamentos utilizados frecuentemente para el tratamiento de la migraña (en forma de ergotamina, dihidroergotamina, etc.) junto con los IP y con efavirenz. Esta asociación genera un aumento en las concentraciones plasmáticas de los ergotamínicos, pudiendo generar ergotismo.

\section{Estatinas}

La estatinas son fármacos de uso frecuente en los pacientes con infección por VIH. Se deberá evitar la asociación de los IP con la lovastatina y simvastatina debido al significativo aumento de las concentraciones plasmáticas de estas últimas. Por otro lado, atorvastatina y rosuvastatina aumentan sus niveles plasmáticos frente al uso concomitante de IP pero en menor medida que lovastatina y simvastatina. Si bien no está contraindicada su asociación, estas estatinas se deberán utilizar con precaución, en las dosis más bajas posibles e ir titulando según respuesta lipídica. Alternativas para usar con IP son fluvastatina y pravastatina (excepto esta última con darunavir/ritonavir, donde deberá utilizarse la menor dosis posible de pravastatina). Con efavirenz, disminuyen los niveles de atorvastatina, lovastatina, simvastatina y pravastatina. Se deberá ajustar la dosis de estatina de acuerdo a la respuesta lipídica.

\section{Anticonvulsivantes}

También existen importantes interacciones con los distintos anticonvulsivantes. Para el caso de carbamazepina, sus niveles plasmáticos aumentan con la coadministración de IP reforzados, así como disminuyen los niveles de IP. Se deberán considerar alternativas a la carbamazepina o monitorizar niveles del anticonvulsivante así como la respuesta virológica. En cuanto a fenitoína, la asociación a IP disminuye los niveles de fenitoína y del IP utilizado. Se deberán considerar alternativas para el anticonvulsivante o monitorizar sus niveles junto con una estricta evaluación de la respuesta virológica. Otros anticonvulsivantes también presentan interacciones con los IP, por lo que se deberá revisar posibles asociaciones según cada paciente en particular. En el caso de los INNTR, su co-administración con anticonvulsivantes también presenta interacciones, como por ejemplo, tanto efavirenz como nevirapina presentan una disminución de sus niveles plasmáticos al asociarlos con carbamazepina o con fenitoína, así como también disminuyen las concentraciones plasmáticas de los anticonvulsivantes. En estos casos se deberán considerar alternativas o realizar un monitoreo estricto de concentraciones séricas de anticonvulsivantes y de la respuesta virológica.

Nota del Editor de sección: En general, la mayor parte de las interacciones farmacológicas están descritas con los anticonvulsivantes antiguos, metabolizados a nivel del citocromos P 450 (carbamazepina, fenobarbital, fenitoína y primidona). No se ha demostrado interacción clínicamente relevante entre efavirenz y ácido valproico. Entre los nuevos anticonvulsivantes, hay descritas interacciones relevantes para lamotrigina y topiramato, por lo que se prefiere el uso de levetiracetam, gabapentina o pregabalina dado que no poseen interacciones relevantes con la TARV (Siddiqi O, Birbeck G L. Safe treatment of seizures in the setting of HIV/AIDS. Curr Treat Options Neurol 2013; 15: 529-43).

\section{Terapia hormonal anticonceptiva}

Es importante trabajar en conjunto con los ginecólogos al indicar el tratamiento de anticoncepción, debido a que 
las interacciones son múltiples entre los IP o los INNTR y los anticonceptivos orales (ACO). Esto es muy importante ya que puede disminuir el efecto de la anticoncepción, con las consecuencias de un embarazo no deseado y con ello, transmisión vertical del VIH.

En el caso de los IP, éstos interactúan con los ACO que contienen etinilestradiol (EE). Las concentraciones del ACO pueden disminuir entre 19 y 48\% según el IP utilizado. El IP de menor interacción con estos ACOs es atazanavir/ritonavir. Sólo en esos casos, es posible utilizar ACO siempre que éste contenga al menos $35 \mu \mathrm{g}$ de EE. A las pacientes que utilizan atazanavir no reforzado, se les puede indicar ACO que contengan un mínimo de $30 \mu \mathrm{g}$ de EE. Para el resto de los IP, se sugiere elegir un ACO alternativo (ej. que contenga progestágeno solo) asociado a métodos de barrera adicionales u otros métodos como implantes subcutáneos con progestágeno, inyección trimestral de acetato de medroxiprogesterona o dispositivo intrauterino con levonorgestre ${ }^{23,63}$.

También se observan interacciones entre algunos IP (lopinavir/ritonavir, fosamprenavir/ritonavir y darunavir/ ritonavir) con la noretisterona, compuesto que se encuentra en algunos ACO y en anticonceptivos de inyección mensual.

Para el caso de los INNTR, el AUC de los ACOs que contienen EE y noretisterona disminuye en $20 \%$ con la asociación a nevirapina, por lo que se recomienda indicar alternativas o métodos adicionales de anticoncepción ${ }^{23,63}$.

Con efavirenz, disminuye en $83 \%$ el AUC del levonorgestrel sistémico (indicado como ACO) y en $64 \%$ del norelgestromin (parches anticonceptivos), por lo que se deben considerar alternativas. También existiría una disminución de la concentración mínima, máxima y vida media del levonorgestrel indicado en dosis de anticoncepción de emergencia cuando se co-administra con efavirenz. Algunos autores sugieren aumentar la dosis al doble $(1,5 \mathrm{mg})$ del levonorgestrel ${ }^{64}$.

Para etravirina $^{23,65}$ y rilpivirina ${ }^{23}$, los niveles plasmáticos de los ACOs que contienen EE aumentan entre 15 y $20 \%$. En estos casos es posible indicarlos y no requerirán de un ajuste de dosis.

Con maraviroc, un estudio no mostró interacciones con co-administración de ACOs combinados (EE/levonorgestrel) en voluntarias sanas ${ }^{66}$.

En cuanto a raltegravir, un estudio de Anderson y cols. ${ }^{67}$, mostró una mínima disminución en la concentración mínima y AUC de ACOs combinados (EE/ norgestimato) con la co-administración de raltegravir, sin ser de importancia clínica.

\section{Antiácidos}

Será importante tener en consideración que la mayoría de las interacciones de antiácidos ocurren con atazanavir dentro de la familia de los IP. El uso de inhibidores de bomba de protones (IBP) no se recomienda en pacientes que se encuentran con atazanavir no reforzado debido a la disminución de sus niveles plasmáticos. En caso de ser muy necesario su uso, el paciente deberá recibir atazanavir con refuerzo de ritonavir. Además la dosis de IBP no podrá superar el equivalente a $20 \mathrm{mg}$ de omeprazol al día. Ambos medicamentos habrá que indicarlos con una separación de 12 horas entre ellos. En cuanto a los antagonistas de receptor $\mathrm{H} 2$, éstos disminuyen los niveles plasmáticos de atazanavir con y sin refuerzo. En los casos de usuarios de atazanavir reforzado, se podrá utilizar un máximo equivalente a $40 \mathrm{mg}$ cada 12 horas de famotidina en pacientes vírgenes a TARV y $20 \mathrm{mg}$ cada 12 horas en experimentados, indicando el atazanavir/ritonavir 10 horas después de la administración del antagonista H2. Para los usuarios de atazanavir no reforzado, se podrá indicar una dosis máxima equivalente a famotidina $20 \mathrm{mg}$ al día, indicando el atazanavir al menos dos horas antes o 10 horas después de la administración del antagonista H2. Para los usuarios de fosamprenavir, se sugiere administrar éste al menos 10 horas posterior a la ingestión del antagonista H2 y reforzarlo con ritonavir. Por último, en cuanto a los antiácidos, éstos podrían disminuir los niveles de algunos IP. Para atazanavir, atazanavir/ritonavir y tipranavir/ritonavir, se deberán indicar éstos al menos dos horas antes o 1 hora después de la administración del antiácido. En el caso del fosamprenavir, se sugiere administrar éste en forma simultánea o al menos dos horas antes o una hora después de la administración del antiácido. No existen interacciones importantes entre antiácidos y los INNTR efavirenz o nevirapina.

\section{Regímenes no recomendados como terapia inicial en pacientes vírgenes a tratamiento ${ }^{23}$}

- Combinación de dos o tres INTR: debido a su menor eficacia virológica comprobada.

- Didanosina en asociación con lamivudina o emtricitabina: debido a su menor eficacia virológica.

- Didanosina asociada a tenofovir: por su alta tasa de falla virológica y producción de mutaciones de resistencia.

- Estavudina: debido a sus toxicidades.

- Fosamprenavir no reforzado: por su menor potencia que el uso del fármaco reforzado y debido a que la falla virológica con fosamprenavir no reforzado puede seleccionar mutaciones de resistencia para darunavir.

- Atazanavir no reforzado: por la mayor emergencia de resistencia a atazanavir (Ver: refuerzo de IP con un segundo fármaco).

- Enfuvirtide: sólo hay experiencia clínica en pacientes experimentados a terapia. 


\section{Refuerzo del inhibidor de proteasa con un segundo fármaco}

Los IP son rápidamente metabolizados por el citocromo P450 3A. Esto genera una inactivación del fármaco que impide que éste alcance concentraciones plasmáticas eficaces, como es el caso de lopinavir, darunavir, tipranavir, o saquinavir, los cuales deberán indicarse siempre reforzados con ritonavir. Con un refuerzo con ritonavir, el fármaco logra alcanzar los niveles plasmáticos adecuados para lograr su efectividad clínica.

Diferente es el caso de atazanavir y fosamprenavir, en los cuales la indicación de refuerzo estará determinada por distintos factores.

Para atazanavir, se ha demostrado la necesidad de reforzar con ritonavir en los pacientes vírgenes a terapia y en aquellos que han fallado previamente a terapia con IP, ya que con cargas virales detectables, atazanavir no reforzado presenta concentraciones variables en el plasma y pueden caer bajo el umbral mínimo de actividad ${ }^{69}$. Esto genera falla en el tratamiento debido a una mayor emergencia de resistencia ${ }^{70}$.

Otros escenarios donde atazanavir deberá reforzarse es el caso de las mujeres embarazadas. Las recomendaciones actuales sugieren que atazanavir/ritonavir es una alternativa para su uso en mujeres embarazadas; sin embargo, éste nunca deberá ser prescrito sin el refuerzo de ritonavir. En el segundo y tercer trimestre, la dosis de esta combinación deberá aumentarse a atazanavir $400 \mathrm{mg} /$ día asociado a ritonavir $100 \mathrm{mg}$ /día debido a que su biodisponibilidad podría reducirse en este período ${ }^{69}$.

En relación al uso concomitante con otros fármacos, la asociación con tenofovir mostró que este último disminuye en $40 \%$ la concentración plasmática mínima de atazana$\operatorname{vir}^{71}$. Sin embargo, nuevos estudios farmacocinéticos ${ }^{72,73}$ han sugerido que la co-administración con tenofovir no explicaría la reducción en las concentraciones de atazanavir. En la impresión del autor, mientras no se dispongan de mayores estudios, es recomendable utilizar el atazanavir reforzado con ritonavir en presencia de tenofovir.

Atazanavir se absorbe mejor con ph gástrico ácido. Por esta razón, la co-administración de IBP u otros antiácidos puede disminuir hasta en $60 \%$ la exposición a atazanavir en plasma llevando a una posible falla virológica. Por esta razón, atazanavir en estos casos deberá ser indicado con refuerzo de ritonavir. En el apartado de interacciones se explicó con detalle las interacciones entre atazanavir e IBP, antagonistas de receptor $\mathrm{H} 2$ y antiácidos. Se entregan alternativas y recomendaciones de uso.

Por otro lado, es posible suspender el ritonavir, manteniendo atazanavir no reforzado en forma segura a pacientes que han alcanzado supresión virológica y que por alguna razón, ya sea por efectos adversos o deseo de simplificación de terapia, se desea eliminar el ritonavir. El estudio ARIES ${ }^{74}$ enroló más de 500 pacientes con infección por VIH vírgenes a tratamiento. Todos los pacientes usaron atazanavir/ritonavir asociado a abacavir/lamivudina. Luego de una fase de inducción de 36 semanas y habiendo alcanzado cargas virales $<50$ copias ARN/ml, se asignaron en forma aleatoria en dos grupos. Un grupo se mantuvo en el mismo régimen mientras en el segundo grupo se eliminó el refuerzo de ritonavir. A la semana $48 ; 86 \%$ y $81 \%$ de los pacientes mantenía cargas virales $<50$ copias ARN/ml en el grupo atazanavir y atazanavir/ ritonavir respectivamente, cumpliendo criterios de no inferioridad para la rama de pacientes en que se suspendió el refuerzo. Estos hallazgos se mantuvieron a la semana 144. No se observaron mutaciones de resistencia en el grupo sin ritonavir. En cuanto a seguridad, se observó que el perfil lipídico del grupo atazanavir no reforzado mejoraba y disminuía el porcentaje de pacientes con hiperbilirrubinemia. Estudios aleatorios más pequeños y otros observacionales confirman estos resultados ${ }^{75,76}$.

Para fosamprenavir es posible usar el fármaco sin refuerzo, en pacientes vírgenes a la terapia, pero indicando el doble de la dosis de fosamprenavir $(1.400 \mathrm{mg}$ cada $12 \mathrm{~h}$ ); sin embargo, en pacientes experimentados, siempre se deberá reforzar con ritonavir.

\section{Pruebas a realizar previo a la utilización de TARV}

De los fármacos utilizados en primera línea como TARV, abacavir requiere de la realización de una prueba de tamizaje previo a su uso.

Con el uso de abacavir, existe un riesgo de 5 a $8 \%$ de presentarse una reacción grave de hipersensibilidad. Esta reacción es una enfermedad multiorgánica que consta de fiebre, rash, síntomas constitucionales, gastrointestinales y respiratorios. Ocurre en promedio a la sexta semana del inicio del fármaco y aumenta en gravedad con las dosis continuas. En caso de presentar alguno de estos síntomas, abacavir se debe discontinuar inmediatamente. Una re-exposición a abacavir está contraindicada, ya que esto puede producir una nueva reacción más grave y potencialmente fatal. Este efecto adverso se asocia con la presencia del alelo HLA-B*5701. En Chile, un estudio publicado por Poggi y cols., mostró 4,7\% de positividad para este alelo en la población general ${ }^{77}$.

El tamizaje de HLA-B*5701 muestra que su presencia se asocia a un valor predictor positivo de 47,9\% (33,3$62,8 \%)$ y un valor predictor negativo de $100 \%(99,5-$ $100 \%$ ) para desarrollar reacción de hipersensibilidad ${ }^{78}$.

Por esta razón, abacavir no debe ser indicado con una prueba positiva para HLA- B*5701. En caso de no tener disponibilidad de realizar la prueba, puede ser razonable iniciar abacavir con consejería adecuada al paciente y monitoreo de posibles efectos adversos ${ }^{23}$. 


\section{Biodisponibilidad de los fármacos de la TARV}

Debido al aumento de su biodisponibilidad, ciertos fármacos deberán ser indicados junto con comidas. Estos son: etravirina, rilpivirina, atazanavir, atazanavir/ritonavir, darunavir/ritonavir, saquinavir/ritonavir. Contrario es el caso de efavirenz, el cual, para disminuir los efectos secundarios, se recomienda indicar en la noche y alejado por dos horas de las comidas.

Tabla 4. Ajuste de dosis de ARVs según velocidad de filtración glomerular ${ }^{68}$

\begin{tabular}{|c|c|c|}
\hline Necesidad de ajuste & \multicolumn{2}{|c|}{ Anti-retroviral } \\
\hline No requiere ajuste & \multicolumn{2}{|c|}{$\begin{array}{l}\text { Abacavir, IP, INNTR, raltegravir, enfuvirtide. } \\
\text { Excepción: NO se recomienda administración de atazanavir en } \\
\text { pacientes sometidos a hemodiálisis }{ }^{79}\end{array}$} \\
\hline \multirow[t]{7}{*}{ Disminuir dosis según $\mathrm{Cl} \mathrm{Cr}$} & ARV & VFG $(\mathrm{ml} / \mathrm{min})$ \\
\hline & Didanosina & $\begin{array}{l}\mathrm{Cl} \mathrm{Cr} \text { 10-50: } 200 \mathrm{mg} / \mathrm{d} \\
\mathrm{Cl} \mathrm{Cr}<10: \\
<60 \mathrm{Kg}: 100 \mathrm{mg} / \mathrm{d} \\
>60 \mathrm{Kg}: 150 \mathrm{mg} / \mathrm{d}\end{array}$ \\
\hline & Zidovudina & $\begin{array}{l}\mathrm{Cl} \mathrm{Cr} 10-50: 300 \mathrm{mg} \mathrm{c} / 12 \mathrm{~h} \\
\mathrm{Cl} \mathrm{Cr}<10: 100 \mathrm{mg} \mathrm{c} / 8 \mathrm{~h}\end{array}$ \\
\hline & Lamivudina & $\begin{array}{l}\mathrm{Cl} \mathrm{Cr} 10-50: 50-150 \mathrm{mg} / \mathrm{d} \\
\mathrm{Cl} \mathrm{Cr}<10: 25-50 \mathrm{mg} / \mathrm{d}\end{array}$ \\
\hline & Emtricitabina & $\begin{array}{l}\mathrm{Cl} \mathrm{Cr} 30-50: 200 \mathrm{mg} \mathrm{c} / 48 \mathrm{~h} \\
\mathrm{Cl} \mathrm{Cr} 10-30: 200 \mathrm{mg} \mathrm{c} / 72 \mathrm{~h} \\
\mathrm{Cl} \mathrm{Cr}<10: 200 \mathrm{mg} \mathrm{c} / 96 \mathrm{~h}\end{array}$ \\
\hline & Tenofovir & $\begin{array}{l}\mathrm{Cl} \mathrm{Cr} 30-50: 300 \mathrm{mg} \mathrm{c} / 48 \mathrm{~h} \\
\mathrm{Cl} \mathrm{Cr} 10-30: 300 \mathrm{mg} / 72-96 \mathrm{~h} \\
\mathrm{Cl} \mathrm{Cr}<10: \sin \text { datos }\end{array}$ \\
\hline & Maraviroc & $\mathrm{Cl} \mathrm{Cr}<50:$ sin datos \\
\hline - Contraindicado en hemodiálisis & \multicolumn{2}{|c|}{$\begin{array}{l}\text { - Tenofovir y tenofovir/emtricitabina } \\
\text { - Atazanavir }\end{array}$} \\
\hline
\end{tabular}

VFG: velocidad de filtración glomerular; Cl Cr: Clearance de creatinina.

\section{Tabla 5. Ajuste de dosis en insuficiencia hepática ${ }^{68}$}

$\begin{array}{ll}\text { Anti-retroviral } & \text { Puntuación Child-Pugh y dosis ajustada de ARV } \\ \text { Abacavir } & \begin{array}{l}\text { Child } 5-6 \text { puntos: } 200 \mathrm{mg} \mathrm{c} / 12 \mathrm{~h} \text {, con cautela } \\ \text { Child }>6 \text { puntos: contraindicada }\end{array} \\ \text { Child hasta } 6 \text { puntos: con cautela } \\ \text { Child }>6 \text { puntos: contraindicada }\end{array}$

ARV: antirretroviral.
Por otro lado, los siguientes ARVs pueden ser indicados con o sin comidas; zidovudina/lamivudina, abacavir/ lamivudina, tenofovir/emtricitabina, nevirapina, lopinavir/ ritonavir, fosamprenavir/ritonavir, raltegravir, maraviroc.

\section{Ajuste de dosis en insuficiencia renal}

Para ciertos ARVs, se deberán ajustar las dosis en relación a la velocidad de filtración glomerular (o clearance de creatinina) del paciente.

Los ajustes se realizarán principalmente sobre la familia de los INTR, y dentro de ésta sobre zidovudina, lamivudina, emtricitabina, didanosina y tenofovir. La excepción dentro de esta familia es abacavir, el cual no requiere ajuste (pero debemos indicarlo en forma separada de lamivudina para ajustar esta última). Está contraindicada la administración de tenofovir con clearance de creatinina menor a $10 \mathrm{ml} / \mathrm{min}$ y de tenofovir/emtricitabina en pacientes con clearance de creatinina menor a $30 \mathrm{ml} /$ min (Tabla 4).

No será necesario ajustar las dosis de los INNTR, los IP ni de raltegravir.

\section{Consideraciones en insuficiencia hepática}

En caso de insuficiencia hepática, dentro de la familia de los INTR, sólo abacavir deberá ajustarse según la puntuación de Child-Pugh. En cuanto a la familia de los INNTR, nevirapina se encuentra contraindicada en pacientes con Child-Pugh B o C. Debido a su metabolismo hepático, distintos IP deberán ser ajustados en estos pacientes. Atazanavir y fosamprenavir serán ajustadas sus dosis considerando el nivel Child-Pugh mientras que darunavir y saquinavir no están recomendados en falla hepática grave (Tabla 5).

\section{Conclusiones}

Es fundamental individualizar la terapia en cada paciente que requiera iniciar TARV. Como se describió durante esta revisión, la decisión dependerá de múltiples factores, entre ellos, la ocupación del paciente, sus comorbilidades, su estado virológico e inmunológico.

Es importante explicar la forma y horario de toma de la TARV al paciente, pues ciertos fármacos funcionan con comida, mientras que otros hay que ingerirlos algunas horas separadas de los alimentos.

Se debe revisar cada uno de los medicamentos asociados para evitar las interacciones medicamentosas, así como también explicarle al paciente que previo a cualquier nuevo medicamento indicado se deberá chequear que no haya contraindicación para asociarlo con su TARV. Una forma práctica de evitar complicaciones es explicarle 
al paciente en la primera consulta los medicamentos prohibidos más importantes para esa TARV en particular.

Finalmente, es importante destacar que el éxito de la terapia no sólo dependerá del paciente y su adherencia, sino también del compromiso del médico tratante con su paciente, la dedicación y tiempo que el médico y otros profesionales del equipo tratante le entregue al paciente para explicar con claridad cada uno de estos puntos antes mencionados.

\section{Resumen}

Uno de los principales factores que influyen en el éxito de la terapia anti-retroviral es la adherencia. Sin embargo, existen otros elementos que el médico debe conocer y cumplir de manera de optimizar la eficacia terapéutica. Si bien distintos fármacos anti-retrovirales han sido aprobados para su uso en pacientes con infección por VIH, cada uno de ellos tiene indicaciones y contraindicaciones particulares que habrá que conocer antes de indicarlos en un paciente en particular. La elección del anti-retroviral dependerá de múltiples factores tales como la edad, sexo, co-morbilidades, co-infecciones, estado virológico e inmunológico del paciente, entre otras. Además, es necesario explicar la importancia del cumplimiento de los horarios de administración y las posibles interacciones con otros medicamentos, todos puntos esenciales para obtener un pronóstico favorable de la terapia.

\section{Referencias bibliográficas}

1.- Palella F J Jr., Delaney K M, Moorman A C, Loveless M O, Fuhrer J, Satten G A, et al. Declining morbidity and mortality among patients with advanced human immunodeficieny virus infection. HIV Outpatient Study Investigators. N Engl J Med 1998; 338: 853-60.

2.- Baylor M S, Johann-Liang R. Hepatotoxicity associated with nevirapine use. J Acquir Immune Defic Syndr 2004; 35: 538-9.

3.- Galli M, Veglia F, Angarano G, Santambrogio S, Meneghini E, Gritti F, et al. Gender differences in antiretroviral drugrelated adipose tissue alterations. Women are at higher risk than men and develop particular lipodystrophy patterns. J Acquir Immune Defic Syndr 2003; 34: 58-61.

4.- Brown T T, Qaqish R B. Response to Berg et al. Antiretroviral therapy and the prevalence of osteopenia and osteoporosis: a meta-analytic review. AIDS 2007; 21: 1830-1.

5.- Thiébaut R, Dequae-Merchadou L, Ekouevi D K, Mercié P, Malvy D, Neau D, et al. Incidence and risk factors of severe hypertriglyceridaemia in the era of highly active antiretroviral therapy: the Aquitaine Cohort, France, 1996-99. HIV Med 2001; 2: 84-8.

6.- Bristol-Myers Squibb. Sustiva drug label, 11/30/2010. Disponible en: http://www. accessdata.fda.gov/drugsatfda_docs/ label/2010/021360s024lbl.pdf. (Accedido: 6 de julio de 2012)

7.- Antiretroviral Pregnancy Registry Steering Committee. Antiretroviral pregnancy registry international interim report for 1 Jan 198931 January 2012. Wilmington, NC: Registry Coordinating Center; 2012. Disponible en http:// www.APRegistry.com. Accedido: 1 de febrero de 2013.

8.- Ford N, Mofenson L, Kranzer K, Medu L, Frigati L, Mills E J, et al. Safety of efavirenz in first-trimester of pregnancy: a systematic review and meta-analysis of outcomes from observational cohorts. AIDS 2010; 24: 1461-70.

9.- Brogly S B, Abzug M J, Watts D H, Cunningham C K, Williams P L, Oleske J, et al. Birth defects among children born to human immunodeficiency virus-infected women: pediatric AIDS clinical trials protocols 219 and 219C. Pediatr Infect Dis J 2010; 29: 721-7.

10.- Panel on Treatment of HIV-infected Pregnant Women and Prevention of Perinatal Transmission. Recommendations for use of antiretroviral drugs in pregnant HIV-1- infected women for maternal health and interventions to reduce perinatal HIV transmission in the United States. Disponible en: http://aidsinfo.nih.gov/ contentfiles/lvguidelines/PerinatalGL.pdf. 2013. E4-E7. Accedido: 5 de junio de 2013.

11.- Grabar S, Kousignian I, Sobel A, Le Bras P, Gasnault J, Enel P, et al. Immunologic and clinical responses to highly active antiretroviral therapy over 50 years of age. Results from the French Hospital Database on HIV. AIDS 2004; 18: 2029-38.

12.- Cooper R D, Wiebe N, Smith N, Keiser P, Naicker S, Tonelli M. Systematic review and meta-analysis: renal safety of tenofovir disoproxil fumarate in HIV-infected patients. Clin Infect Dis 2010; 51: 496-505.

13.- Nelson M R, Katlama C, Montaner J S, Cooper D A, Gazzard B, Clotet B, et al. The safety of tenofovir disoproxil fumarate for the treatment of HIV infection in adults: the first 4 years. AIDS 2007, 21: 1273-81.

14.- Young J, Schafer J, Fux C A, Furrer H, Bernasconi E, Vernazza P, et al. Renal function in patients with HIV starting therapy with tenofovir and either efavirenz, lopinavir or atazanavir. AIDS 2012; 26: 567-75.

15.- Goicoechea M, Liu S, Best B, Sun S, Jain S, Kemper C, et al. Greater tenofovir-associated renal function decline with protease inhibitor-based versus nonnucleoside reversetranscriptase inhibitor-based therapy. J Infect Dis 2008; 197: 102-8.
16.- Ryom L, Mocroft A, Kirk O, Worm S W, Kamara D A, Reiss P, et al. Exposure to antiretrovirals (ARVs) and risk of renal impairment among HIV positive persons with normal baseline renal function: the D:A:D study. J Infect Dis 2013; 207: 1359-69.

17.- Stellbrink H J, Orkin C, Arribas J R, Compston J, Gerstoft J, Van Wijngaerden E, et al. Comparison of changes in bone density and turnover with abacavir-lamivudine versus tenofovir-emtricitabine in HIV-infected adults: 48-week results from the ASSERT study. Clin Infect Dis 2010; 51: 963-72.

18.- Duvivier C, Kolta S, Assoumou L, Ghosn J, Rozenberg S, Murphy R L, et al. Greater decrease in bone mineral density with protease inhibitor regimens compared with nonnucleoside reverse transcriptase inhibitor regimens in HIV-1 infected naive patients. AIDS 2009, 23: 817-24.

19.- Sabin C A, Worm S W, Weber R, Reiss P, El-Sadr W, Dabis F, et al. Use of nucleoside reverse transcriptase inhibitors and risk of myocardial infarction in HIV-infected patients enrolled in the D:A:D study: a multi-cohort collaboration. Lancet 2008; 371: 1417-26.

20.- Cruciani M, Zanichelli V, Serpelloni G, Bosco O, Malena M, Mazzi R, et al. Abacavir use and cardiovascular disease events: a metaanalysis of published and unpublished data. AIDS 2011; 25: 1993-2004.

21.- Choi AI, Vittinghoff E, Deeks S G, Weekley C C, Li Y, Shlipak M G. Cardiovascular risks associated with abacavir and tenofovir exposure in HIV-infected persons. AIDS 2011; 25: 1289-98.

22.- Lennox J L, Dejesus E, Berger D S, Lazzarin A, Pollard R B, Ramalho Madruga J V, et al. Raltegravir versus efavirenz regimens in treatment-naive HIV-1-infected patients: 96-week efficacy, durability, subgroup, safety, and metabolic analyses. J Acquir Immune Defic Syndr 2010; 55: 39-48.

23.- Panel on Antiretroviral Guidelines for Adults 
and Adolescents. Guidelines for the use of antiretroviral agents in HIV-1-infected adults and adolescents. Department of Health and Human Services. Disponible: http://aidsinfo. nih.gov/guidelines/June 2013. Accedido: 10 de febrero de 2013.

24.- Graham C S, Baden L R, Yu E, Mrus J M, Carnie J, Heeren T, et al. Influence of human immunodeficiency virus infection on the course of hepatitis $\mathrm{C}$ virus infection: a meta-analysis. Clin Infect Dis 2001; 33: 562-9.

25.- Ghany M G, Strader D B, Thomas D L, Seeff L B. Diagnosis, management, and treatment of hepatitis $\mathrm{C}$ : an update. Hepatology 2009; 49: 1335-74.

26.- Soriano V, Puoti M, Sulkowski M, Cargnel A, Benhamou Y, Peters M, et al. Care of patients coinfected with HIV and hepatitis C virus: 2007 updated recommendations from the HCV-HIV International Panel. AIDS 2007; 21: 1073-89.

27.- European AIDS Clinical Society. Guidelines for the clinical management and treatment of chronic hepatitis $\mathrm{B}$ and $\mathrm{C}$ co-infection in HIV-infected adults (version 6.1). November 2012. Disponible: http://www. europeanaidsclinicalsociety.org/images/stories/ EACS-Pdf/EacsGuidelines-v6.1-2edition.pdf. Accedido: 9 de junio de 2013.

28.- Gane E J, Roberts S K, Stedman C A, Angus P W, Ritchie B, Elston R, et al. Oral combination therapy with a nucleoside polymerase inhibitor (RG7128) and danoprevir for chronic hepatitis $\mathrm{C}$ genotype 1 infection (INFORM-1): a randomized, double-blind, placebo controlled, dose escalation trial. Lancet 2010; 376: 1467-75.

29.- Zeuzem S, Soriano V, Asselah T, Bronowicki J P, Lohse A W, Mullhaupt B et al. Interferon (INF)-free combination treatment with $\mathrm{HCV}$ NS3/4A protease inhibitor BI 201335 and the non-nucleoside NS 5B inhibitor BI 207127 +/ribavirin (R): final results of SOUND-C2 and predictors of response. $63^{\text {rd }}$ Annual Meeting of the American Association for the Study of Liver Diseases, Boston, abstract 232, 2012.

30.- Gane E J, Stedman C A, Hyland R H, Ding X, Svarovskaia E, Symonds W T, et al. Nucleotide polymerase inhibitor sofosbuvir plus ribavirin for hepatitis C. N Engl J Med 2013; 368: 34-44.

31.- Poordad F, Lawitz E, Kowdley K, Cohen D E, Podsadecki T, Siggelkow S, et al. Exploratory study of oral combination antiviral therapy for hepatitis C. N Engl J Med 2013; 368: 45-53.

32.- DeJesus E, Herrera G, Teofilo E, Gerstoft J, Buendía C B, Brand J D, et al. Abacavir versus zidovudine combined with lamivudine and efavirenz, for the treatment of antiretroviralnaive HIV-infected adults. Clin Infect Dis 2004; 39: 1038-46.

33.- Pozniak A L, Gallant J E, DeJesus E, Arribas J R, Gazzard B, Campo R E, et al. Tenofovir disoproxil fumarate, emtricitabine, and efavirenz versus fixed-dose zidovudine/ lamivudine and efavirenz in antiretroviralnaive patients: virologic, immunologic, and morphologic changes-a 96-week analysis. J Acquir Immune Defic Syndr 2006; 43: 535-40.

34.- Sax P E, Tierney C, Collier A C, Daar E, Mollan K, Budhathoki C, et al. Abacavir/ lamivudine versus tenofovir DF/emtricitabine as part of combination regimens for initial treatment of HIV: final results. J Infect Dis 2011; 204: 1191-201.

35.- Smith K Y, Patel P, Fine D, Bellos N, Sloan L, Lackey P, et al. Randomized, double-blind, placebo-matched, multicenter trial of abacavir/ lamivudine or tenofovir/emtricitabine with lopinavir/ritonavir for initial HIV treatment. AIDS 2009; 23: 1547-56.

36.- Riddler S, Haubrich R, DiRienzo G, Peeples L, Powderly W G, Klingman K L, et al. Classsparing regimens for initial treatment of HIV-1 infection. N Engl J Med 2008; 358: 2095-106.

37.- van Leth F, Phanuphak P, Ruxrungtham K, Baraldi E, Miller S, Gazzard B, et al. Comparison of first-line antiretroviral therapy with regimens including nevirapine, efavirenz, or both drugs, plus stavudine and lamivudine: a randomised open-label trial, the 2NN Study. Lancet 2004; 363:1253-63.

38.- Molina J M, Cahn P, Grinsztejn B, Lazzarin A, Mills A, Saag M, et al. Rilpivirine versus efavirenz with tenofovir and emtricitabine in treatment-naive adults infected with HIV-1 (ECHO): a phase 3 randomised double-blind active-controlled trial. Lancet 2011; 378: 238-46.

39.- Katlama C, Haubrich R, Lalezari J, Lazzarin A, Madruga J V, Molina J M, et al. Efficacy and safety of etravirine in treatment-experienced, HIV-1 patients: pooled 48 week analysis of two randomized, controlled trials. AIDS 2009; 23: 2289-300.

40.- Gazzard B, Duvivier C, Zagler C, Castagna A, Hill A, van Delft Y, et al. Phase 2 double-blind, randomized trial of etravirine versus efavirenz in treatment-naive patients: 48 -week results. AIDS 2011; 25: 2249-58.

41.- Molina J M, Andrade-Villanueva J, Echevarría J, Chetchotisakd P, Corral J, David N, et al. Once-daily atazanavir/ritonavir versus twice-daily lopinavir/ritonavir, each in combination with tenofovir and emtricitabine, for management of antiretroviral-naive HIV-1infected patients: 48 week efficacy and safety results of the CASTLE study. Lancet 2008; 372: 646-55.

42.- Molina J M, Andrade-Villanueva J, Echevarría J, Chetchotisakd P, Corral J, David N, et al. Once-daily atazanavir/ritonavir compared with twice-daily lopinavir/ritonavir, each in combination with tenofovir and emtricitabine for management of antiretroviralnaive HIV-1-infected patients: 96-week efficacy and safety results of the CASTLE Study. J

Acquir Immune Defic Syndr 2010; 53: 323-32

43.- Smith K Y, Weinberg W G, DeJesus E, Fischl M A, Liao Q, Ross L L, et al. Fosamprenavir or atazanavir once daily boosted with ritonavir $100 \mathrm{mg}$, plus tenofovir/ emtricitabine, for the initial treatment of HIV infection: 48-week results of ALERT. AIDS Res Ther 2008; $5: 5$.

44.- Eron J Jr., Yeni P, Gathe J Jr., Estrada V, De Jesus E, Staszewski S, et al. for the KLEAN study team. The KLEAN study of fosamprenavir-ritonavir versus lopinavirritonavir, each in combination with abacavirlamivudine, for initial treatment of HIV infection over 48 weeks: a randomised noninferiority trial. Lancet 2006; 368: 476-82.

45.- Pulido F, Estrada V, Baril J G, Loque K, Schewe K, Plettenberg A, et al. Longterm efficacy and safety of fosamprenavir plus ritonavir versus lopinavir/ritonavir in combination with abacavir/lamivudine over 144 weeks. HIV Clin Trials 2009; 10: 76-87.

46.- Walmsley S, Avihingsanon A, Slim J, Ward D J, Ruxrungtham K, Brunetta J, et al. Gemini: a noninferiority study of saquinavir/ ritonavir versus lopinavir/ritonavir as initial HIV-1 therapy in adults. J Acquir Immune Defic Syndr 2009; 50: 367-74.

47.- Ortiz R, Dejesus E, Khanlou H, Voronin E, van Lunzen J, Andrade-Villanueva J, et al. Efficacy and safety of once-daily darunavir/ ritonavir versus lopinavir/ritonavir in treatmentnaive HIV-1-infected patients at week 48 . AIDS 2008; 22: 1389-97.

48.- Ministerio de Salud, Chile. Guía Clínica Síndrome de inmunodeficiencia adquirida VIH/SIDA. Santiago: MINSAL, 2009. Rev Chilena Infectol 2010; 27 (3): 237-76.

48a.-Ministerio de Salud, Chile. Guía Clínica Síndrome de inmunodeficiencia adquirida VIH/SIDA. Santiago: MINSAL, 2009. Rev Chilena Infectol 2010; 27 (4): 361-73.

49.- Lennox J L, DeJesus E, Lazzarin A, Pollard R B, Madruga J V, Berger D S, et al. Safety and efficacy of raltegravir-based versus efavirenz-based combination therapy in treatment-naive patients with HIV-1 infection: a multicentre, double-blind randomised controlled trial. Lancet 2009; 374: 796-806.

50.- Rockstroh J K, De Jesus E, Henry K, Molina J M, Gathe J, Ramanathan S, et al. A randomized, double-blind comparison of coformulated elvitegravir/ cobicistat/emtricitabine/tenofovir DF vs ritonavir-boosted atazanavir plus coformulated emtricitabine and tenofovir DF for initial treatment of HIV-1 infection: analysis of week 96 results. J Acquir Immune Defic Syndr. 2013; 62: 483-6.

51.- Zolopa A, Sax P E, DeJesus E, Mills A, Cohen C, Wohl D, et al. A randomized double- 
blind comparison of coformulated elvitegravir/ cobicistat/emtricitabine/tenofovir disoproxil fumarate versus efavirenz/emtricitabine/ tenofovir disoproxil fumarate for initial treatment of HIV-1 infection: analysis of week 96 results. J Acquir Immune Defic Syndr. 2013; 63: 96-100.

52.- Cooper D A, Heera J, Goodrich J, Tawadrous M, Saag M, DeJesus E, et al. Maraviroc versus efavirenz, both in combination with zidovudine-lamivudine, for the treatment of antiretroviral-naive subjects with CCR5tropic HIV-1 infection. J Infect Dis 2010; 201: 803-13.

53.- Sohler N L, Wong M D, Cunningham W E, Cabral H, Drainoni M L, Cunningham C O. Type and pattern of illicit drug use and access to health care services for HIV-infected people. AIDS Patient Care STDS. 2007; 21 (Suppl 1): S68-S76.

54.- Ceballos ME, Pérez C, Rojas A, Donato P, Huilcamán M, Rivera G, et al. Resultados de la terapia antirretroviral en pacientes con VIH en control en la Pontificia Universidad Católica de chile. Cohorte VIH-PUC. Comunicación oral CO24, XXIX Congreso Chileno de Infectología, Pucón, octubre de 2012.

55.- Hahn J A, Samet J H. Alcohol and HIV disease progression: weighing the evidence. Curr HIV/ AIDS Rep 2010; 7: 226-33.

56.- Chander G, Lau B, Moore R D. Hazardous alcohol use: a risk factor for non-adherence and lack of suppression in HIV infection. J Acquir Immune Defic Syndr 2006; 43: 411-7.

57.- Ghebremichael M, Paintsil E, Ickovics J R, Vlahov D, Schuman P, Boland R et al. Longitudinal association of alcohol use with HIV disease progression and psychological health of women with HIV. AIDS Care 2009; 21: 834-41.

58.- Cook J A, Burke-Miller J K, Cohen M H, Cook R L, Vlahov D, Wilson T E, et al. Crack cocaine, disease progression, and mortality in a multicenter cohort of HIV-1 positive women. AIDS 2008; 22: 1355-63.

59.- Wolff M J, Cortés C P, Shepherd B E, Beltrán C J. Long-term outcomes of a national expanded access program to antiretroviral therapy: the Chilean AIDS cohort. J Acquir Immune Defic Syndr 2010; 55: 368-74.

60.- Dierberg K L, Chaisson R E. Human immunodeficiency virus-associated tuberculosis: update on prevention and treatment. Clin Chest Med 2013; 34: 217-28.

61.- Centers for Disease Control and Prevention. Managing drug interactions in the treatment of HIV-related tuberculosis. December 2007. Disponible: http://www.cdc.gov/tb/TB_HIV_ Drugs/default.htm. Accedido: 10 de junio de 2013.

62.- Grinsztejn B, De Castro N, Arnold V, Veloso V, Morgado M, Pilotto J, et al. A randomized multicentre open-label trial to estimate the efficacy and safety of two doses of raltegravir (RAL) to efavirenz (EFV) for the treatment of HIV-TB co-infected patients: results of the ANRS 12180 Reflate TB trial. Abstract XIX International AIDS Conference, 2012, July 2227. Washington DC, USA.

63.- Robinson J A, Jamshidi R, Burke A E. Contraception for the HIV-positive woman: a review of interactions between hormonal contraception and antiretroviral therapy. Infect Dis Obstet Gynecol. 2012; 2012: 890160

64.- Carten M, Kiser J, Kwara A, MaWhinney S, $\mathrm{Cu}-$ Uvin S. Pharmacokinetic (PK) interactions between the hormonal emergency contraception Plan B (levonorgestrel) and efavirenz (EFV). En Proceedings of the 17th Conference on Retroviruses and Opportunistic Infections, San Francisco, California, USA, February 2010.

65.- Scholler-Gyure M, Kakuda T, Woodfall B, Aharchi F, Peeters M, Vandermeulen K, et al. Effect of steady-state etravirine on the pharmacokinetics and pharmacodynamics of ethinylestradiol and norethindrone. Contraception 2009; 80: 44-52.

66.- Abel S, Russell D, Whitlock L A, Ridgway C E, Muirhead G J. Effect of maraviroc on the pharmacokinetics of midazolam, lamivudine/ zidovudine and ethinyloestradiol/levonorgestrel in healthy volunteers. Br J Clin Pharmacol 2008; 65 Suppl 1: 19-26.

67.- Anderson M S, Hanley W D, Moreau A R, Jin B, Bieberdorf F A, Kost J T, et al. Effect of raltegravir on estradiol and norgestimate plasma pharmacokinetics following oral contraceptive administration in healthy women. Br J Clin Pharmacol 2011; 71: 616-20.

68.- Saag M, Chambers H, Eliopoulus G, Gilbert D, Moellering R. The Sanford Guide to HIV/AIDS Therapy. 19th edition. Sperryville, U.S.A. 2011; 183-90.

69.- Foca E, Ripamonti D, Motta D, Torti C. Unboosted atazanavir for treatment of HIV infection rationale and recommendations for use. Drugs 2012; 72: 1161-73.

70.- Malan D R, Krantz E, David N, Wirtz V, Hammond J, Mc Grath D. Efficacy and safety of atazanavir, with or without ritonavir, as part of once-daily highly active antiretroviral therapy regimens in antiretroviral-naive patients. J Acquir Immune Defic Syndr 2008; 47: 161-7.

71.- No authors listed. Atazanavir (Reyataz): new recommendations if combined with tenofovir (Viread) - and warning on Viagra, Cialis, and Levitra. AIDS Treat News 2004; (399): 5

72.- Calcagno A, Bonora S, Tettoni M C, D’Avolio A, Perri G D, Lanzafame M, et al. Tenofovir coadministration is not associated with lower unboosted atazanavir plasma exposure in the clinical setting. J Acquir Immune Defic Syndr 2009; 52: 431-2.

73.- von Hentig N, Dauer B, Haberl A, Klauke S, Lutz T, Staszewski S, et al. Tenofovir comedication does not impair the steadystate pharmacokinetics of ritonavir-boosted atazanavir in HIV-1-infected adults. Eur J Clin Pharmacol 2007; 63: 935-40.

74.- Squires K E, Young B, DeJesus E, Bellos N, Murphy D, Zhao H H, et al. Similar efficacy and tolerability of atazanavir compared with atazanavir/ritonavir, each with abacavir/ lamivudine after initial suppression with abacavir/lamivudine plus ritonavir-boosted atazanavir in HIV-infected patients. AIDS 2010, 24: $2019-27$

75.- Ghosn J, Carosi G, Moreno S, Poksrovsky V, Lazzarin A, Pialoux G, et al. Unboosted atazanavir based therapy maintains control of HIV type-1 replication as effectively as a ritonavir-boosted regimen. Antivir Ther 2010; 15: 993-1002.

76.- Hocqueloux L, Le Moal G, De la Tribonnie 're X, Prazuck T, Plainchamp D, Choisy P, et al. Maintenance therapy with unboosted atazanavir is comparable to boosted strategy in experienced patients, even with tenofovir: a multicentre propensityadjusted cohort. 6th IAS Conference on HIV Pathogenesis, Treatment and Prevention; 2011 Jul 17-20; Rome Astract CDB359.

77.- Poggi H, Vera A, Lagos M, Solari S, Rodríguez P L, Pérez C M. HLA-B*5701 frequency in Chilean HIV-infected patients and in general population. Braz J Infect Dis 2010; 14: $510-2$.

78.- Mallal S, Phillips E, Carosi G, Molina J M, Workman C, Tomazic J, et al. HLA-B*5701 screening for hypersensitivity to abacavir. $\mathrm{N}$ Engl J Med 2008; 358: 568-79.

79.- ATAZANAVIR. Summary of product characteristics. BRISTOL-MYERS SQUIBB S.R.L. European Medical Agency. Agosto 2012: 1-170. Disponible en: http://www.emea.europa. eu/ema/. Accedido: 7 de febrero de 2013. 